\title{
Xanthone, benzophenone and bioflavonoid accumulation in Cyclopia genistoides (L.) Vent. (honeybush) shoot cultures grown on membrane rafts and in a temporary immersion system
}

\author{
Adam Kokotkiewicz • Adam Bucinski • \\ Maria Luczkiewicz
}

Received: 8 May 2014/ Accepted: 28 July 2014/Published online: 7 August 2014

(c) The Author(s) 2014. This article is published with open access at Springerlink.com

\begin{abstract}
In vitro shoots of the commercially important South-African legume Cyclopia genistoides were grown in different liquid culture systems [membrane rafts (MR) and a temporary immersion bioreactor (TIB)] and evaluated for the accumulation of phenolic secondary metabolites. The major constituents of the investigated cultures were medicinally relevant xanthones [mangiferin (M) and isomangiferin (IM)] and benzophenone derivatives [iriflophenone 3- $C$ - $\beta$-glucoside (IG)]. The highest concentrations of M, IM and IG in MR-grown shoots were 1,843.59, 712.02 and $594.29 \mathrm{mg}$ $100 \mathrm{~g}^{-1}$ dry wt, respectively. Bioreactor cultivation provided higher peak concentrations of $M(2,622.70 \mathrm{mg}$ $100 \mathrm{~g}^{-1}$ dry wt), IM (757.40 mg $100 \mathrm{~g}^{-1}$ dry wt) and IG (648.30 mg $100 \mathrm{~g}^{-1}$ dry wt) which corresponded to the respective productivities of $5.48,1.58$ and $3.04 \mathrm{mg} \mathrm{l}^{-1} \mathrm{~d}^{-1}$. The results indicate that TIB cultures of $C$. genistoides may be utilized as an alternative source of the above constituents, particularly IM and IG, which are relatively expensive and so far hardly available from commercial sources.
\end{abstract}

Keywords Benzophenones - Mangiferin - Isomangiferin · Isoflavones · Membrane rafts - Temporary immersion bioreactor

A. Kokotkiewicz · M. Luczkiewicz ( $₫)$

Department of Pharmacognosy, Faculty of Pharmacy, Medical University of Gdansk, Al. Gen. J. Hallera 107, 80-416 Gdansk, Poland

e-mail: mlucz@gumed.edu.pl

\footnotetext{
A. Bucinski

Department of Biopharmacy, Faculty of Pharmacy, Ludwik Rydygier Collegium Medicum in Bydgoszcz, Nicolaus Copernicus University in Torun, Ul. dr A. Jurasza 2, 85-089 Bydgoszcz, Poland
}

\author{
Abbreviations \\ CG Calycosin 7-O- $\beta$-glucoside \\ DW Dry weight \\ FG Formononetin 7-O- $\beta$-glucoside \\ Gi Growth index \\ $\mathrm{H} \quad$ Hesperidin \\ IBA Indole-3-butyric acid \\ IG Iriflophenone 3 - $C$ - $\beta$-glucoside \\ IM Isomangiferin \\ 2iP 2-isopentenyladenine \\ M Mangiferin \\ MG Maclurin 3- $C$ - $\beta$-glucoside \\ MR Membrane raft \\ PG Pseudobaptigenin 7-O- $\beta$-glucoside \\ SH Schenk \& Hildebrandt \\ TDZ Thidiazuron \\ TIB Temporary immersion bioreactor
}

Cyclopia genistoides (L.) Vent. (Fabaceae) is an endemic, South-African legume, native to fynbos shrublands of the Western Cape Province. Together with other representatives of the genus, $C$. genistoides is used to manufacture the 'honeybush' herbal tea, recognized for distinctive, honeylike flavour (Joubert et al. 2011). C. genistoides is characterized by exceptionally high content of the xanthone mangiferin (M), present in the amounts exceeding 5 and $10 \%$ dry weight (DW) in the whole herb and leaves, respectively (Kokotkiewicz et al. 2012, 2013b; Joubert et al. 2014). This compound is known to exhibit a number of biological activities including anti-inflammatory, antidiabetic and chemopreventive (Matkowski et al. 2013), thus contributing to the health-promoting properties of the honeybush. Two other important $C$. genistoides constituents, 
isomangiferin (IM) and iriflophenone-3- $C$ - $\beta$-glucoside (IG) constituting ca. 2 and $1 \%$ DW, respectively (Kokotkiewicz et al. 2012, 2013b; Joubert et al. 2014), have so far not been extensively studied with respect to their biological effects. However, both compounds were recently shown to be potent antioxidants (Malherbe et al. 2014) and also demonstrated strong pro-apoptotic activity on rheumatoid arthritis synoviocytes (Kokotkiewicz et al. 2013b). Moreover, IG and IGcontaining Cyclopia extracts were shown to inhibit adipogenesis in 3T3-L1 cell line (Dudhia et al. 2013).

The established biological effects of xanthone and benzophenone constituents of $C$. genistoides indicate that the discussed plant has potential value in the management of diseases such as rheumatoid arthritis, diabetes and obesity. Unfortunately, Cyclopia currently faces the risk of over exploitation due to high demand from overseas markets. Occasional droughts and fynbos fires additionally limit the availability of plant material (Joubert et al. 2011). These problems can be overcome by establishing in vitro cultures of $C$. genistoides, which could serve as a renewable source of xanthone and benzophenone derivatives for biological activity studies. In the presented work, in vitro shoot cultures of $C$. genistoides were adapted for the growth in liquid culture systems [membrane rafts (MR) and temporary immersion bioreactor (TIB)], and evaluated for the production of phenolic secondary metabolites. For the authors knowledge, this is the first report on establishing bioreactor cultures for the production of M, IM and IG.

The source of plant material were $C$. genistoides microshoot cultures, maintained on solidified $(0.6 \% \mathrm{w} / \mathrm{v}$ agar) Schenk \& Hildebrandt ( $\mathrm{SH})$ medium supplemented with $3.0 \% \mathrm{w} / \mathrm{v}$ sucrose, $9.84 \mu \mathrm{M}$ 2-isopentenyladenine (2iP) and $1.0 \mu \mathrm{M}$ thidiazuron (TDZ). These cultures are deposited in the Higher Plants Biotechnology Laboratory at the Department of Pharmacognosy, Medical University of Gdansk, Poland, and is available to other researchers. As demonstrated in previous study (Kokotkiewicz et al. 2012), C. genistoides microshoots accumulated xanthones ( $\mathrm{M}$ and $\mathrm{IM})$, flavanone hesperidin $(\mathrm{H})$, as well as three isoflavone glucosides [7-O- $\beta$-glucosides of calycosin (CG), formononetin (CG) and pseudobaptigenin (PG)], absent in intact plant material but otherwise characteristic for undifferentiated cultures of Cyclopia (Kokotkiewicz et al. 2013a, 2014). However, since the current research focused on establishing an in vitro source of xanthones and benzophenones, the experiments were conducted using $\mathrm{SH}$ medium enriched with indole-3-butyric acid (IBA), which was previously shown to enhance the accumulation of phenolics typical for field-grown plants (Kokotkiewicz et al. 2012). Unfortunately, attempts to obtain shaker cultures proved unsuccessful, probably due to high sensitivity of the investigated biomass to mechanical stress and/or explant browning, triggered by excessive contact with the growth medium (Kokotkiewicz et al. 2012). Therefore, it was decided to grow the shoots using the MR system, which provides the advantages of liquid cultures (enhanced exchange of nutrient and metabolites between plant tissues and the growth medium) without exerting mechanical stress on the explants (Vágner et al. 2005).

For the experiment, $2.5 \mathrm{~g}$ portions of shoot primordia (taken on $30 \mathrm{~d}$ of the growth cycle) were transferred into polycarbonate 'Magenta' vessels, equipped with 'LifeRaft' cell/tissue support system (floating raft with bottom made of hydrophilic microporous membrane which prevents plant tissues from sinking and coming into direct contact with the medium). The growth containers were subsequently filled with $50 \mathrm{ml}$ of the SH medium, supplemented with $4.92 \mu \mathrm{M}$ IBA and $1.5 \% \mathrm{w} / \mathrm{v}$ sucrose. 'Magenta' vessels, membrane rafts and culture reagents were obtained from Sigma-Aldrich (St. Louis, US-MO). The cultures were maintained at $24 \pm 1{ }^{\circ} \mathrm{C}$ under continuous light $\left(88 \pm 8 \mu \mathrm{mol} \mathrm{m}{ }^{-2} \mathrm{~s}^{-1}\right.$, TLD $35 \mathrm{~W}$ white fluorescent tubes, Philips, Amsterdam, the Netherlands). Biomasses and media samples were collected in 4-day intervals. The length of growth period ( $60 \mathrm{~d}$ ) was set based on the results of the previous studies concerning $C$. genistoides micropropagation which showed substantial increase in xanthone levels in explants elongated for 2 months on IBA-supplemented medium (Kokotkiewicz et al. 2012). The harvested shoots were evaluated for polyphenol content according to the previously described methodology (Kokotkiewicz et al. 2012, 2013a).

As presented in Fig. 1a-c, during the first 24 days of the experiment MR-grown shoots showed intensive growth, accompanied by a marked increase in xanthones content. The peak concentrations of M and IM were achieved on 36 $\mathrm{d}$ which corresponded to the stationary phase, and decreased shortly afterwards. Maximum concentration of IG preceded those of M and IM (Fig. 1b-d), suggesting the incorporation of IG into xanthone derivatives. However, such translocation has so far not been reported in other species (Joubert et al. 2014) and establishing whether the reaction(s) occurs in Cyclopia would require further studies. Except for CG, whose concentration showed an initial increase until $12 \mathrm{~d}$ of experiment, isoflavones content gradually decreased during the culture period (Fig. 1f-h), whereas the concentrations of $\mathrm{H}$ remained fairly unchanged, and relatively low, in the course of experiment (Fig. 1e). None of the investigated compounds was detected in the media samples.

In general, the results confirmed our previous finding which linked the removal of exogenous cytokinins from the medium, and the accompanying changes in auxin/ cytokinin balance and shoot morphology (elongation and reduction of hyperhydricity), with increased accumulation of phenolics typical for $C$. genistoides intact plant 
Fig. 1 Changes in growth index (a) and concentrations of phenolic secondary metabolites (b-h) in membrane raft-grown C. genistoides shoot cultures. Growth indices were calculated using the formula:

$\mathrm{Gi}=\left(\left[\mathrm{G}_{\mathrm{n}}-\mathrm{G}_{0}\right] / \mathrm{G}_{0}\right) \times 100 \%$ where $G_{n}$ and $G_{0}$ are fresh weights on the nth and 0 day of experiment, respectively. Different letters indicate significant differences among means $(\mathrm{n}=3)$ based on Tukey's range test $(p \leq 0.05)$
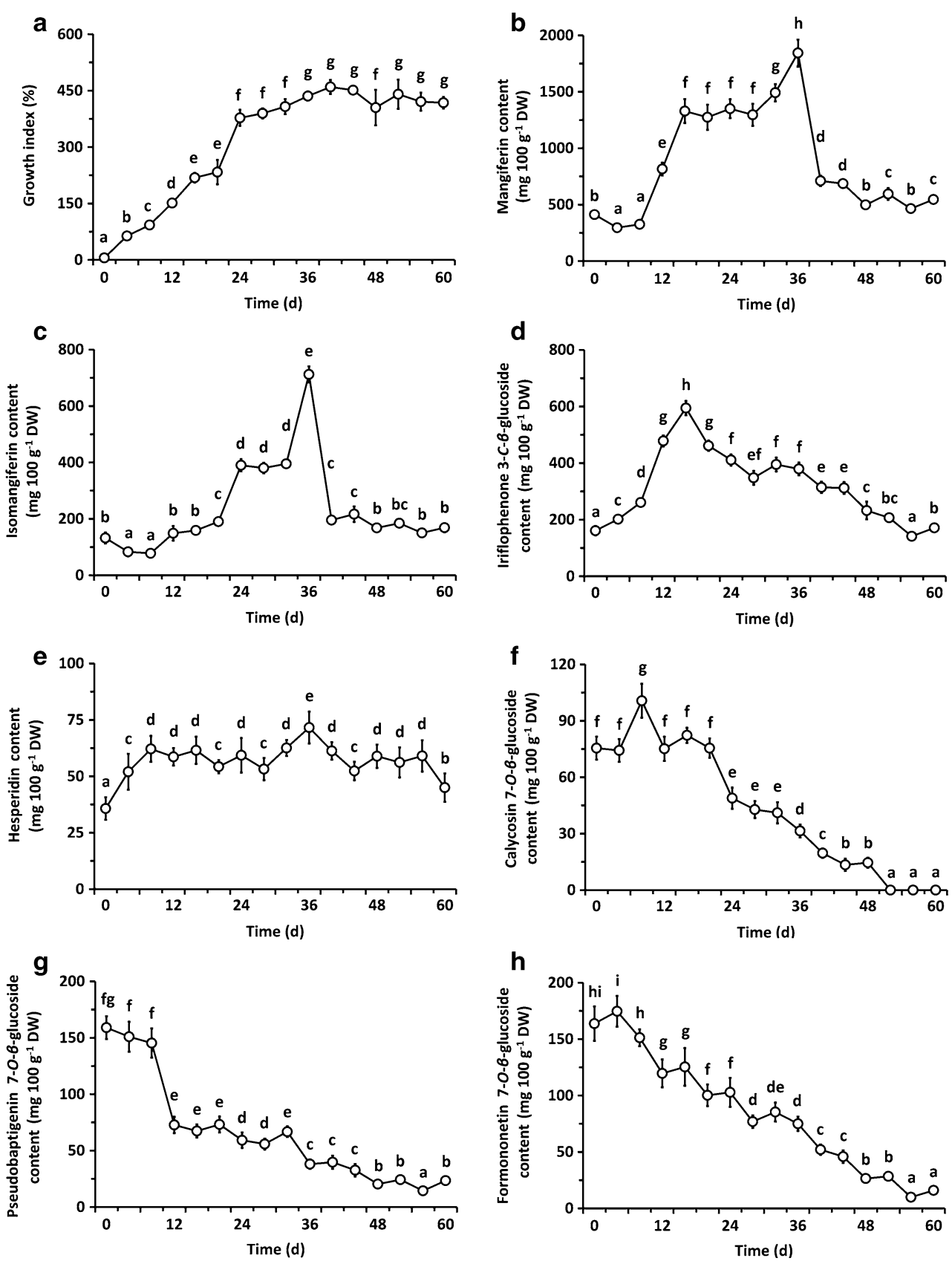

(Kokotkiewicz et al. 2012). Interestingly, other studies concerning in vitro cultures of mangiferin-producing plants (e.g. Gentiana spp.) did not show the inhibitory effect of the purine-type cytokinin (6-benzyladenine) on xanthone biosynthesis (Menković et al. 2000; Dević et al. 2006). Therefore, decreased biosynthesis of mangiferin in $C$. genistoides microshoots grown on the cytokinin-supplemented medium may be either speciesspecific, or result from the presence of TDZ, the phenylurea derivative not included in the cited Gentiana studies.
Further part of the research focused on establishing bioreactor cultures of $C$. genistoides. Given the previously reported sensitivity of the investigated culture to mechanical stress, it was decided that the shoots will be grown in the temporary immersion system. TIBs were successfully used for maintaining in vitro cultures of numerous medicinally-relevant plants, serving as a source of cardiac glycosides (Pérez-Alonso et al. 2012), anticancer alkaloids (Sankar-Thomas and Lieberei 2011) and bioflavonoids (Zobayed et al. 2004). The details of the structure and operation mode of the TIB employed for the experiments 
Fig. 2 The temporary immersion bioreactor (TIB) employed for the maintenance of $C$. genistoides shoot cultures. a System overview, b shoots grown for $32 \mathrm{~d}$ and $\mathbf{c}$ schematic diagram of the bioreactor: 1 glass culture vessel $(150 \mathrm{~mm}$ id, $200 \mathrm{~mm} \mathrm{~h}, 300 \mathrm{ml}$ working volume), $1 a$ stainless steel basket for biomass immobilization $(120 \times 100 \mathrm{~mm}, 8 \mathrm{~mm}$ mesh $)$, positioned to provide $1 \mathrm{~cm}$ shoots submersion depth during the immersion phase), 2 magnetic stirrer, 3 time controller, 4,5 peristaltic pumps, 6 air pump, 7 air prefilter, 8 flowmeter, 9 air humidifier, 10,11 air sterilisation filters, 12 medium reservoir $(1,000 \mathrm{ml}$ total medium volume). White and black arrows indicate the direction of air and medium flow, respectively. The bioreactor was operated in 45/45 min immersion cycles. Aeration $(0.4 \mathrm{vvm})$ and mixing $(38 \times 8 \mathrm{~mm}$ cylindrical stirrer bar, $200 \mathrm{rpm}$ ) were provided only during the immersion phase a

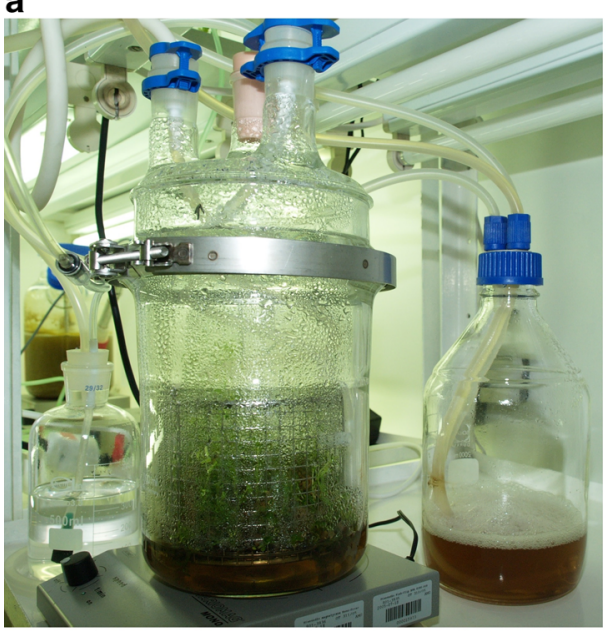

b

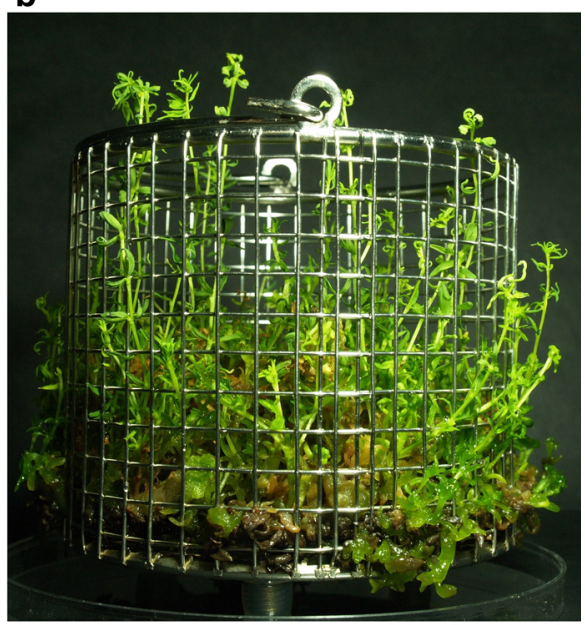

C

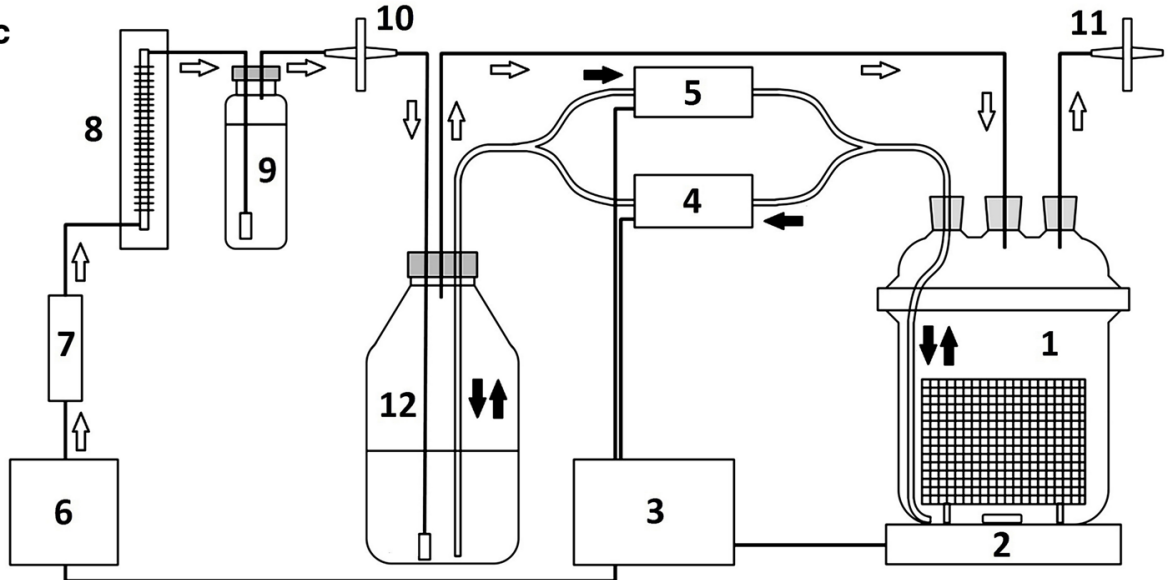

were presented in Fig. 2. In order to minimise mechanical stress the shoot explants were subjected to, the growth medium was aerated only in the reservoir while low-speed magnetic stirrer provided gentle mixing during the immersion phase. The bioreactor was inoculated in the same manner as MR cultures (1:20 shoots:medium ratio). According to the results of previous studies (Kokotkiewicz et al. 2012), the experiment time was set to 32 and 60 days.

As presented in Fig. 3a, TIB-grown shoots showed slightly higher growth rates than cultures maintained using MR system. M, IM and H concentrations exceeded those recorded in MR-grown shoots, but were achieved only on the 60th day of experiment (Fig. 3b, c, f). Regardless of the harvest time, IG content of TIB-grown shoots was higher than that of MR cultures (Fig. 3d). Interestingly, bioreactor- grown shoots also accumulated low amounts of the benzophenone MG (Fig. 3e), previously identified in C. genistoides intact plant material (Kokotkiewicz et al. 2013b) but absent in MR cultures of the examined plant. Isoflavone glucosides (CG, PG and FG) were present in small amounts only in 32-d TIB culture.

The productivities of $C$. genistoides in vitro systems in terms of three major phenolic derivatives (M, IM anf IG) were included in Table 1. In general, it was shown that compared to MR cultures, TIB-grown shoots maintain the ability to produce significantly higher amounts of the investigated compounds during a 60-day-long experiment. On the other hand, 32-daylong cultivation significantly favoured the production of M, IM and IG in MR cultures, as well as IG in the TIB system. The calculated productivities of IM and IG, which are much less abundant than mangiferin (and consequently, more expensive), may be interesting from a practical perspective. The obtained results indicate that $C$. genistoides shoot cultures could be successfully maintained in commercially available and easily multipliable temporary-immersion (RITA) (Zobayed et al. 2004) or raft (Growtek) bioreactor systems (Sharma et al. 2011), and utilized for the production of biologically active xanthone and benzophenone derivatives. 

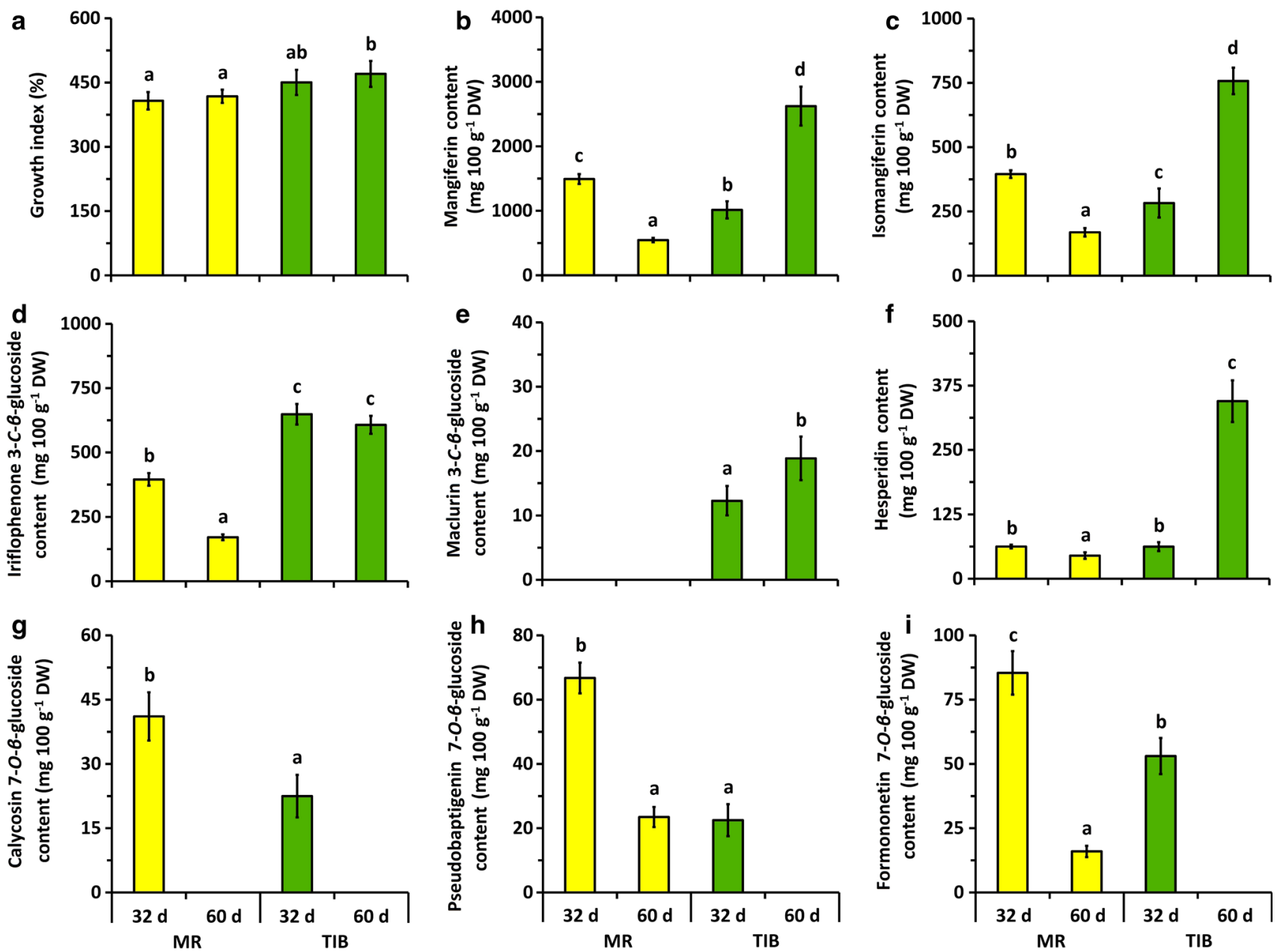

Fig. 3 The comparison of growth indices (a) and concentrations of phenolic secondary metabolites (b-i) in $C$. genistoides shoot cultures grown on membrane rafts (MR) or in the temporary immersion

bioreactor (TIB). Different letters indicate significant differences among means $(n=3$ and $n=2$ for MR and TIB experiments, respectively) based on Tukey's range test $(p \leq 0.05)$

Table 1 Productivities of xanthone and benzophenone derivatives in C. genistoides shoot cultures grown on membrane rafts (MR) or in temporary immersion bioreactor (TIB) for 32 or 60 days

\begin{tabular}{lllll}
\hline Growth system used & Day of the experiment & \multicolumn{3}{l}{ Productivity $\left(\mathrm{mg} \mathrm{l}^{-1} \mathrm{~d}^{-1}\right)^{\mathrm{a}}$} \\
\cline { 3 - 5 } & & $\mathrm{M}$ & IM & $1.65 \pm 0.08^{\mathrm{b}}$ \\
\hline Membrane rafts & 32 & $6.74 \pm 0.40^{\mathrm{c}}$ & $1.78 \pm 0.13^{\mathrm{c}}$ \\
Temporary immersion bioreactor & 60 & $1.09 \pm 0.07^{\mathrm{a}}$ & $0.34 \pm 0.03^{\mathrm{a}}$ & $0.34 \pm 0.02^{\mathrm{a}}$ \\
& 32 & $4.76 \pm 0.66^{\mathrm{b}}$ & $1.33 \pm 0.28^{\mathrm{b}}$ & $3.04 \pm 0.21^{\mathrm{d}}$ \\
& 60 & $5.48 \pm 0.66^{\mathrm{b}}$ & $1.58 \pm 0.11^{\mathrm{b}}$ & $1.27 \pm 0.08^{\mathrm{b}}$ \\
\hline
\end{tabular}

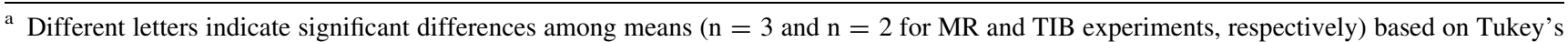
range test $(p \leq 0.05)$, data comparison in columns

Acknowledgments This study was supported by grant no. NN302 041936 from the Polish Ministry of Education and Science.

Conflict of interest The authors also declare that they have no conflict of interest. The described experiments are of non-commercial nature and were conducted for scientific purposes only.

Open Access This article is distributed under the terms of the Creative Commons Attribution License which permits any use, distribution, and reproduction in any medium, provided the original author(s) and the source are credited.

\section{References}

Dević M, Momčilović I, Krstić D, Maksimović V, Konjewić R (2006) In vitro multiplication of willow gentian (Gentiana asclepiadea 
L.) and the production of gentiopicrine and mangiferin. Phyton 46:45-54

Dudhia Z, Louw J, Muller C, Joubert E, de Beer D, Kinnear C, Pheiffer C (2013) Cyclopia maculata and Cyclopia subternata (honeybush tea) inhibits adipogenesis in 3T3-L1 pre-adipocytes. Phytomedicine 20:401-408. doi:10.1016/j.phymed.2012.12.002

Joubert E, Joubert ME, Bester C, de Beer D, De Lange JH (2011) Honeybush (Cyclopia spp.): from local cottage industry to global markets - the catalytic and supporting role of research. S Afr J Bot 77:887-907. doi:10.1016/j.sajb.2011.05.014

Joubert E, de Beer D, Hernández I, Munné-Bosch S (2014) Accumulation of mangiferin, isomangiferin, iriflophenone-3-C$\beta$-glucoside and hesperidin in honeybush (Cyclopia genistoides Vent.) in response to harvest time, harvest interval and seed source. Ind Crops Prod 56:74-82. doi:10.1016/j.indcrop.2014.02. 030

Kokotkiewicz A, Luczkiewicz M, Hering A, Ochocka R, Gorynski K, Bucinski A, Sowinski P (2012) Micropropagation of Cyclopia genistoides, an endemic South African plant of economic importance. Z Naturforsch 67c:65-76

Kokotkiewicz A, Luczkiewicz M, Kowalski W, Badura A, Piekus N, Bucinski A (2013a) Isoflavone production in Cyclopia subternata Vogel (honeybush) suspension cultures grown in shake flasks and stirred-tank bioreactor. Appl Microbiol Biotechnol 97:8467-8477. doi:10.1007/s00253-013-5099-z

Kokotkiewicz A, Luczkiewicz M, Pawlowska J, Luczkiewicz P, Sowinski P, Witkowski J, Bryl E, Bucinski A (2013b) Isolation of xanthone and benzophenone derivatives from Cyclopia genistoides (L.) Vent. (honeybush) and their pro-apoptotic activity on synoviocytes from patients with rheumatoid arthritis. Fitoterapia 90:199-208. doi:10.1016/j.fitote.2013.07.020

Kokotkiewicz A, Bucinski A, Luczkiewicz M (2014) Light and temperature conditions affect bioflavonoid accumulation in callus cultures of Cyclopia subternata Vogel (honeybush). Plant Cell Tiss Organ Cult. doi:10.1007/s11240-014-0502-8

Malherbe CJ, Willenburg E, de Beer D, Bonnet SL, van der Westhuizen JH, Joubert E (2014) Iriflophenone-3- $C$-glucoside from Cyclopia genistoides: isolation and quantitative comparison of antioxidant capacity with mangiferin and isomangiferin using on-line HPLC antioxidant assays. J Chromatogr B 951-952: 164-171. doi:10.1016/j.jchromb.2014.01.038

Matkowski A, Kuś P, Góralska E, Woźniak D (2013) Mangiferin-a bioactive xanthonoid, not only from mango and not just antioxidant. Mini-Rev Med Chem 13:439-455. doi:10.2174/ 1389557511313030011

Menković N, Šavikin-Fodulović K, Momcilović I, Grubišić D (2000) Quantitative determination of secoiridoid and $\gamma$-pyrone compounds in Gentiana lutea cultured in vitro. Planta Med 66:96-98

Pérez-Alonso N, Capote A, Gerth A, Jiménez E (2012) Increased cardenolides production by elicitation of Digitalis lanata shoots cultured in temporary immersion systems. Plant Cell Tiss Organ Cult 110:153-162. doi:10.1007/s11240-012-0139-4

Sankar-Thomas YD, Lieberei R (2011) Camptothecin accumulation in various organ cultures of Camptotheca acuminata Decne grown in different culture systems. Plant Cell Tiss Organ Cult 106:445-454. doi:10.1007/s11240-011-9942-6

Sharma V, Goyal S, Ramawat KG (2011) Increased puerarin biosynthesis during in vitro shoot formation in Pueraria tuberosa grown in growtek bioreactor with aeration. Physiol Mol Biol Plants 17:87-92. doi:10.1007/s12298-011-0049-7

Vágner M, Vondráková Z, Fischerová L, Opatrná J (2005) Norway spruce somatic embryogenesis: membrane rafts as a compromise between liquid and solidified media. In: Hvoslef-Eide AK, Preil $\mathrm{W}$ (eds) Liquid culture systems for in vitro plant propagation. Springer, Dordrecht, pp 295-302. doi:10.1007/1-4020-3200-5 21

Zobayed SMA, Murch SJ, Rupasinghe HPV, de Boer JG, Glickman BW, Saxena PK (2004) Optimized system for biomass production, chemical characterization and evaluation of chemopreventive properties of Scutellaria baicalensis Georgi. Plant Sci 167:439-446. doi:10.1016/j.plantsci.2004.04.022 